risk (born at 30 and 31 weeks' GA, not SGA, without severe morbidities), risks of DLD were higher when mothers had less than high school versus tertiary education (RR word combination: 2.2 (95\% CI: 1.5; 3.3); RR expressive vocabulary: 1.5 (95\% CI: 1.1; 2.0)). Among children with higher perinatal risk (lower GA, SGA and severe morbidities), maternal education was not associated with DLD.

Conclusion Maternal education was associated with language development only among VPT children with low perinatal risk. The interaction of social factors with perinatal risk may explain contradictory findings in previous studies.

\section{P54 PARENTAL RATING OF FOLLOW-UP CARE FOR THEIR CHILDREN IN A EUROPEAN COHORT OF VERY PRETERM BIRTHS}

${ }^{1,2}$ AV Seppanen*, ${ }^{3}$ E Draper, ${ }^{1,2} \mathrm{R}$ El Rafei, ${ }^{4,5} \mathrm{~L}$ Toome, ${ }^{6} \mathrm{M}$ Cuttini, ${ }^{7} \mathrm{~S}$ Petrou, ${ }^{8} \mathrm{H}$ Barros, 1J Zeitlin, on behalf of SHIPS Research Group. 'French National Institute of Health and Medical Research (INSERM), Paris, France; ${ }^{2}$ Sorbonne University, Paris, France; ${ }^{3}$ Department of Health Sciences, University of Leicester, Leicester, UK: ${ }^{4}$ Clinic of Pediatrics, Tallinn Children's Hospital, Tallinn, Estonia; ${ }^{5}$ Department of Pediatrics, Institute of Clinical Medicine, University of Tartu, Tartu, Estonia; ${ }^{6}$ Clinical Care and Management Innovation Research Area, Bambino Gesu Children's Hospital, Rome, Italy; 'Department of Economics, University of Warwick, Coventry, UK; ${ }^{8}$ Institute of Public Health University of Porto, Porto, Portugal

\subsection{6/jech-2019-SSMabstracts.205}

Background Infants born very preterm are at risk of developing multiple health and developmental problems. Because the prognosis of each individual child is unknown at discharge, follow-up is essential for identifying health needs early, enabling timely intervention and coordinating health services from multiple providers. Despite its recognized importance, there have been few evaluations of follow-up - in particular among parents, whose involvement is crucial for successful follow-up. This study investigated how parents rate their very preterm children's follow-up care in Europe.

Methods The data come from the Screening to improve Health In very Preterm infantS (SHIPS) study. It followed up the area-based EPICE cohort, which included all births before 32 weeks' gestation from all maternity units in 19 regions from 11 European countries in 2011/12. Perinatal data were abstracted from medical records and socioeconomic and child health data were collected with parent-report questionnaires at 2 and 5 years. At 5 years, parents rated the follow-up care received for their child's prematurity (poor, fair, good, excellent) and provided suggestions for improvement as free-text comments. We assessed poor and fair ratings and associated factors, including country, sociodemographic characteristics, perinatal characteristics and current health and developmental problems in STATA 14.0 using $\chi^{2}$; tests and logistic regression models. We grouped free-text comments by themes and described them by country.

Results Questionnaires were filled in for 3414 children (51\% response rate), by mothers (84\%), fathers (14\%) and other caretakers. 93\% reported receiving follow-up care. Few (13.7\%) judged follow-up care to be poor or fair, but this varied from $<10 \%$ in France and the Netherlands to $>20 \%$ in Denmark and Poland $(\mathrm{p}<0.001)$. Higher maternal education was related with more dissatisfaction $(p<0.01)$. Poor/fair ratings were highest when children had diagnosed health problems, especially cerebral palsy $(32.2 \%)$, and developmental delay $(28.5 \%)$. After adjustment for diagnoses, perinatal characteristics were not significantly related to care ratings.
Common themes from free-text comments (1032 responses) included the need for longer-term follow-up, focusing on more than physical health and lack of knowledge about prematurity among general practitioners. Some themes were mentioned more frequently in some countries such as waiting times (Poland), lack of coordination (Sweden) and length of maternity leave (Portugal).

Conclusion Dissatisfaction with follow-up care was low overall, but it was higher among those most reliant on health services. Many common themes emerged from parent comments despite geographic heterogeneity. Further research is needed to understand differences in reported satisfaction between countries and by maternal educational level.

\section{P55 DESIGNING EXPERT HEARINGS: HOW TO USE DELIBERATIVE RESEARCH METHODS FOR PUBLIC HEALTH EVIDENCE}

1J South*, 'J Woodall, ${ }^{1} \mathrm{M}$ Gamsu, ${ }^{2} \mathrm{P}$ Branney, ${ }^{3} \mathrm{R}$ Newton, ${ }^{1} \mathrm{AM}$ Bagnall. ${ }^{1}$ School of Health and Community Studies, Leeds Beckett University, Leeds, UK; ${ }^{2}$ Division of Psychology, University of Bradford, Bradford, UK; ${ }^{3}$ NHS England, NHS, Leeds, UK

\subsection{6/jech-2019-SSMabstracts.206}

Background Deliberative methods, such as citizen juries, are used in public policy as a form of democratic engagement. Because they stimulate dialogue between actors and allow consideration of different evidence sources including lived experience, such methods are often used to examine contentious or complex issues. There are few examples of where deliberative methods have been used in population health research. Deliberative methods have the potential to improve the policy relevance of research and hence its impact - particularly where evidence might be contested. This presentation will consider the application of deliberative expert hearings as a method of collecting, interpreting and validating public health evidence and discuss critical design choices that shape implementation.

Methods Three case studies are presented to illustrate use of expert hearings and methodological choices:

i. A series of expert hearings conducted for a qualitative study into lay people's roles in public health

ii. An expert hearing bringing together stakeholders from across the criminal justice system as part of a systematic review on peer education

iii. Two hearings conducted to test the meaning and application of evidence collected as part of a What Works Centre programme.

The main features were incorporation of democratic principles of deliberation; valuing diverse evidence sources including experiential evidence; in-depth exploration of complex and contested issues; enabling actors to question evidence; testing arguments. Design and sampling choices created the conditions for deliberation on agreed topics. In each case, qualitative data from presentations and discussions were collected and later analysed with a framework, plotting different perspectives on a matrix.

Discussion These studies enabled us to build experience of expert hearings as a research method. In each case, we documented design issues and reflections. This has generated a set of design choices:

Development of research questions - by researchers or with stakeholders; 
Sampling strategy to select participants and their roles in a hearing - expert witness, inquiry panel, facilitator or audience; The type of evidence presented - scientific, professional or lay;

What counts as data and how deliberation is recorded;

Quality of deliberation and whether a consensus is achieved;

Ethical issues about the public nature of deliberation.

Conclusion Deliberative methods are under-utilised as a research method in public health; however, they require attention to design issues to secure genuine deliberation on a topic. This set of design choices will support researchers in generating and testing evidence through an expert hearing.

\section{P56 CHARACTERISTICS ASSOCIATED WITH CARDIOVASCULAR MULTIMORBIDITY IN UK WOMEN AGED 50-64 YEARS: CROSS-SECTIONAL ANALYSIS OF THE MILLION WOMEN STUDY}

JW Suh*, BJ Cairns, FL Wright. Nuffield Department of Population Health, University of Oxford, Oxford, UK

\subsection{6/jech-2019-SSMabstracts.207}

Background Multimorbidity, the co-occurrence of two or more chronic conditions in one person, is more common in women than in men. It is associated with lower life expectancy, lower quality of life, and greater use of health services compared to single diseases. Research on risk factors for cardiovascular multimorbidity (CVM), that is, having multiple cardiovascular diseases (CVDs), has been limited. We aimed to identify potential risk factors for CVM in women.

Methods The Million Women Study is a cohort of 1.3 million women aged 50-64 years, recruited in England and Scotland in 1996-2001 through NHS screening centres. Participants completed an extensive health and lifestyle questionnaire. Record linkage with NHS databases provided hospital admission records. Twenty chronic CVDs were selected based on clinical importance and number of records, primarily from chapter IX of the International Classification of Diseases v10. Characteristics were compared between women with 0,1 or $2+$ CVDs recorded in self-reports and hospital admissions up to recruitment, adjusting for five age categories (referent: 56-59 years).

Results Among 1,272,020 women, 0.7\% $(\mathrm{n}=8463)$ had CVM and $5.3 \%(66,805)$ had one CVD. The most common CVDs were ischaemic heart disease (4.7\%), stroke (1.2\%), atrial fibrillation (0.2\%), and venous thromboembolism (0.2\%); among those with CVM, 85\% had ischaemic heart disease and 54\% had stroke. Women with CVM were older and, after adjustment for age, were more likely to have common cardiovascular risk factors. In women with no CVDs and those with CVM, respectively: $19.2 \%(95 \%$ CI 19.2-19.3) and $25.1 \%$ (24.1-26.0) were current smokers; 39.4\% (39.4-39.6) and $22.7 \%(21.8-23.7)$ did strenuous physical activity at least once a week; mean BMI was $26.2(26.1-26.2)$ and 28.1 (28.0-28.2). Women with CVM were substantially more likely to be in the most deprived fifth and have no educational qualifications. There was little association between CVM and reproductive factors, although women who had ever breastfed were slightly less likely to have CVM. Women with no CVDs were much less likely to report treatment for diabetes, hypertension, and high cholesterol than those with CVM; $15.0 \%$ (14.9-15.1) and 43.9\% (42.9-45.0), respectively, were being treated for hypertension.
Conclusion Age-adjusted prevalence of CVM in UK women was associated with behavioural and socioeconomic characteristics, and with treatment for major cardiovascular risk factors, but largely not with reproductive factors. This cross-sectional study could not assess potential for reverse causation or confounding by other factors, and future prospective analyses will contribute to better understanding of these relationships.

\section{P57 TESTING THE IMPACT OF IAPT ON HEALTHCARE COSTS AND EMPLOYMENT: A STEP-WEDGE DESIGN}

${ }^{1} \mathrm{~V}$ Toffolutti ${ }^{*},{ }^{2}$ Thames Valley, ${ }^{3}$ Oxford Academic Network, ${ }^{4} \mathrm{M}$ McKee, ${ }^{1,5} \mathrm{D}$ Stuckler, ${ }^{3,6} \mathrm{D}$ Clark. ${ }^{1}$ Dondena Centre, Bocconi University, Milan, Italy; ${ }^{2}$ Thames Valley Clinical Commissioning Groups, Thames Valley Clinical Commissioning Groups, UK; ${ }^{3}$ Oxford Academic Network, University of Oxford, Oxford, The UK; ${ }^{4}$ Department of Public Health and Policy, London School of Hygiene and Tropical Medicine, London, UK; ${ }^{5}$ Department of Social and Political Science, Bocconi University, Milan, Italy; ${ }^{6}$ Department of Experimental Psychology, University of Oxford, Oxford, UK

\subsection{6/jech-2019-SSMabstracts.208}

Background According to the World Health Organization (WHO), depression is ranked as the single largest contributor to global disability. The Improving Access to Psychological Therapies (IAPT) programme is a large-scale initiative that aims to greatly increase the availability of NICE recommended psychological treatment for depression and anxiety disorders within the National Health Service in England. This study evaluates whether IAPT reduces healthcare utilization and associated health care costs and increases transition into employment.

Methods Gradual implantation of a stepped-wedge design of two cohorts covering 500 patients with depression and/or anxiety and comorbid long-term physical health conditions from three areas in Thames Valley (Berkshire, Oxfordshire and Buckinghamshire) for the period March 2017 - August 2017.

Results The wedge study findings showed a decrease in cost $£ 345$ total pp for 3 months so about $£ 115$ a month difference between the two cohorts. Results also showed a decrease by about 4.61[95\% CI: $-5.56,-3.66]$ (6.64 [95\% CI: -7.67, 5.61]) $[0.76$ [95\% CI: $-1.22,-0.30]]$ points per person in the GAD7 (PHQ9) [WASAS]. With respect to employment, results to find a job for those who were unemployment by about 29.9\% [95\% CI: 1.37-52.4], the marginal effects corresponds to 6.28 percentage points [95\% CI: 2.19-12.3].

Conclusion IAPT treatment was associated with a significant decline in secondary care costs and significant increase in the probability to find a job for unemployed patients.

Supported by an ERC Grant 313590-HRES. Also funded by Wellcome Trust.

\section{P58 ASSESSMENT OF CARDIOVASCULAR RISK IN A SLUM POPULATION IN KENYA: USE OF WORLD HEALTH ORGANIZATION/INTERNATIONAL SOCIETY OF HYPERTENSION (WHO/ISH) RISK PREDICTION CHARTS}

${ }^{1} \mathrm{~A}$ Vusirikala*, ${ }^{2} \mathrm{~F}$ Wekesah, ${ }^{2} \mathrm{C}$ Kyobutungi, ${ }^{1} \mathrm{O}$ Oyebode. 'Warwick Medical School, University of Warwick, Coventry, UK; ${ }^{2}$ African Population and Health Research Centre, (APHRC), Nairobi, Kenya

\subsection{6/jech-2019-SSMabstracts.209}

Background Although cardiovascular disease (CVD) is of growing importance in low- and middle-income countries (LMICs), 